\title{
Four-dimensional optical manipulation of colloidal particles
}

\author{
Peter John Rodrigo, Vincent Ricardo Daria, and Jesper Glückstad ${ }^{\text {a) }}$ \\ Optics and Plasma Research Department, Ris $\phi$ National Laboratory, DK-4000 Roskilde, Denmark
}

(Received 8 October 2004; accepted 12 January 2005; published online 11 February 2005)

\begin{abstract}
We transform a $\mathrm{TEM}_{00}$ laser mode into multiple counterpropagating optical traps to achieve four-dimensional simultaneous manipulation of multiple particles. Efficient synthesis and dynamic control of the counterpropagating-beam traps is carried out via the generalized phase contrast method, and a spatial polarization-encoding scheme. Our experiments genuinely demonstrate real-time, interactive particle-position control for forming arbitrary volumetric constellations and complex three-dimensional trajectories of multiple particles. This opens up doors for cross-disciplinary cutting-edge research in various fields. (c) 2005 American Institute of Physics.

[DOI: $10.1063 / 1.1866646]$
\end{abstract}

Sculpted light fields have advanced our ability to manipulate colloidal aggregates and brought unique opportunities for fundamental and applied science. ${ }^{1}$ From a physical stance, tailored optical potentials were used to investigate underlying mechanisms of optically bound matter, ${ }^{2,3}$ stochastic resonance in Brownian particles, ${ }^{4}$ and light-matter angular momentum transfer. ${ }^{5}$ Of equivalent significance are the applications of optical manipulation for powering colloidal microfluidic machines ${ }^{6}$ and fractionating mixtures through optical lattices. ${ }^{7,8}$ Tailored optical landscapes have also been applied to organize matter. Arrays of microscopic objects have been patterned in one dimension, ${ }^{9}$ two dimensions (2D),${ }^{10,11}$ and three dimensions (3D). ${ }^{12}$ In particular, material engineers have seen the potentials of optical manipulation in synthesizing advanced materials. This is substantiated in recent works showing the complementary role of optical trapping with laser-initiated photopolymerization for the construction and gelling of permanent particle arrays from linear to crystal-like structures. ${ }^{13,14}$ Aside from the ability to form predefined structures in 3D, however, a number of applications, including those of biological relevance, would significantly gain from the power of being able to arbitrarily adjust the relative positions of particle aggregates over a full volume and in a manner that satisfies real human response time.

With the advent of spatial light modulators (SLM), rapid developments boosting the degree of control for optically trapped particles have been seen in two techniques based on diffractive optics $(\mathrm{DO})^{12,13}$ and the generalized phase contrast (GPC) method. ${ }^{11,15-17}$ Recently, we have shown the suitability of the GPC approach for direct and user-interactive manipulation of a colony of particles and living cells simultaneously trapped and manipulated in a two-dimensional plane with no surface contact. ${ }^{18}$ Here, we implement the GPC method to demonstrate optical micromanipulation of colloidal particles independently trapped and real-time manipulated throughout a large volume. This is achieved using a light-efficient configuration based on multiple counterpropagating-beam traps. Using a lossless polarization scheme, ${ }^{19}$ the axial positions of each individually trapped particle are varied by changing the relative powers of orthogonally polarized counterpropagating beams. This is accomplished by using a spatially addressable polarization

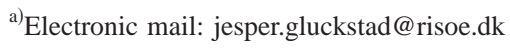

modulator, which effectively separates the command on the relative strengths of individual pairs of opposing beams forming each trap. Since the transverse intensity patterns used to construct the trapping configurations are synthesized by the GPC method, transverse trap positions and dynamics are inherently reconfigurable in real time. The combined result enables, four-dimensional (4D) optical manipulation of a plurality of simultaneously trapped microscopic particles.

The instrumentation of our 4D optical manipulation system is shown in Fig. 1. An expanded TEM $_{00}$ mode from a near-infrared laser is illuminating a phase-only SLM. Upon reflection, the approximately planar incident wave front acquires a 2D phase distribution encoded on the SLM frontpanel surface. The programmable phase function, described by $\phi(u, v)$, is represented as a spatial pattern of up to 256 grey levels on a designated computer graphic monitor. A reasonably linear relation is set between the SLM phase and monitor grey level. For reconfigurable patterns of phase $\phi(u, v)$ in our system implementation, binary grey levels corresponding to phases $\phi=0$ and $\phi=\pi$, suffice. This implies that faster types of SLMs based on ferroelectric liquid crystals, quantum well devices, or microelectromechanical systems-mirrors can be applied. By utilizing a GPC-based light projection setup, the binary phase $\phi(u, v)$ is efficiently

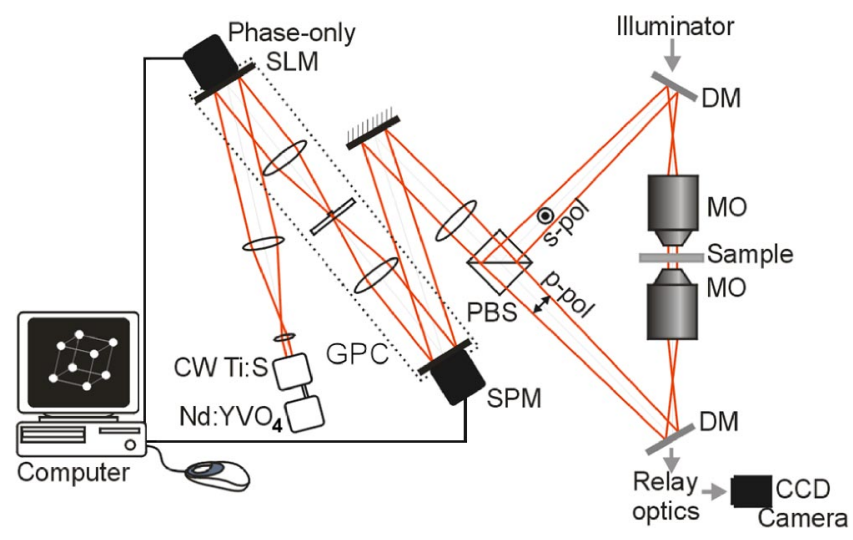

FIG. 1. (Color online) Experimental setup for implementing 4D optical manipulation. cw Ti:S, continuous-wave titanium:sapphire (wavelength $=830 \mathrm{~nm} ;$ maximum power $=1.5 \mathrm{~W}) ; \mathrm{Nd}: \mathrm{YVO}_{4}$, neodymium:yttriumvanadate (wavelength $=532 \mathrm{~nm}$ ); GPC, generalized phase contrast system; SLM, spatial light modulator; SPM, spatial polarization modulator; PBS, polarizing beam splitter; $\mathrm{MO}$, microscope objective $[\times 60$; numerical aperature (NA) $=0.85]$; DM, dichroic mirror. 


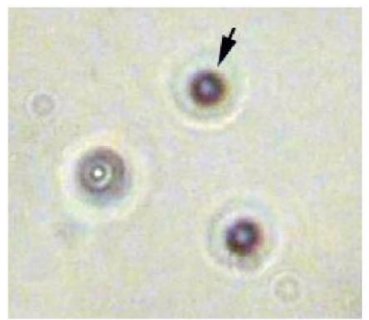

$0 s$

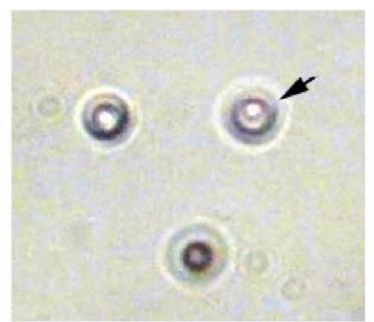

$2 \mathrm{~s}$

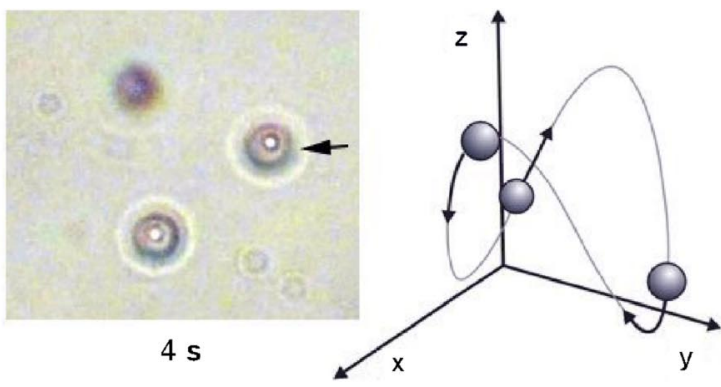

FIG. 2. (Color online) Defining 3D trajectories for multiple $(N=3)$ optically trapped polystyrene spheres (diameter $=3 \mu \mathrm{m})$. The trace of the $3 \mathrm{D}$ path is shown, right (see Ref. 23).

converted into a high-contrast intensity pattern $I\left(u^{\prime}, v^{\prime}\right){ }^{20-22}$ The intensity pattern is then projected onto the front-panel of a spatial polarization modulator (SPM). Here, the process is described by

$$
\begin{aligned}
\phi(u, v) & =\pi \sum_{n=1}^{N} f_{n}\left(u-u_{n}, v-v_{n}\right) \stackrel{\mathrm{GPC}}{\rightarrow} I\left(u^{\prime}, v^{\prime}\right) \\
& \approx I_{0} \sum_{n=1}^{N} f_{n}\left(u^{\prime}-u_{n}^{\prime}, v^{\prime}-v_{n}^{\prime}\right),
\end{aligned}
$$

where $f_{n}(u, v)=f_{n}\left(u^{\prime} / s, v^{\prime} / s\right)$ (i.e., $s$ magnification 4- $f$ imaging) denotes the desired geometrical feature of the $n$th trap centered at $\left(u_{n}^{\prime}, v_{n}^{\prime}\right)$ with the condition that no two traps overlap. For brevity, we also assume a Dirac-delta type imaging point-spread function in our description so that the same functional form of $I\left(u^{\prime}, v^{\prime}\right)$ acceptably represents the projected pattern to our sample plane. Thus, for the traps used (in general, $f_{n}(u, v)$ sets an arbitrary pattern for each trap) with top-hat cross-sectional intensity profiles, we can write $f_{n}(u, v)=\operatorname{circ}\left(\sqrt{u^{2}+v^{2}} / a_{n}\right)$, where $a_{n}$ defines the crosssectional radius, which is individually adjustable for each trap. Standard optimization of the GPC process, described in our previous work, ${ }^{22}$ is followed to attain high photon efficiency and optimal visibility in generating $I\left(u^{\prime}, v^{\prime}\right)$, i.e., $I_{0}$ in Eq. (1) is maximized.

At this point, it is worth to discuss the various modes under which the system can operate. To enumerate these modes, we need to describe the reflection that occurs on the SPM. The SPM modulates the incident linearly polarized field $E\left(u^{\prime}, v^{\prime}\right) \hat{\boldsymbol{e}}_{v}$ whose dot product with its complex conjugate is proportional to $I\left(u^{\prime}, v^{\prime}\right)$. A computer encodes a unique
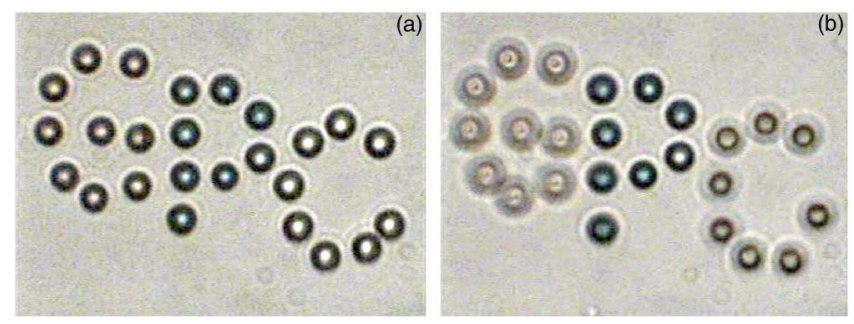

FIG. 3. (Color online) User-coordinated patterning of commercially dyed polystyrene spheres (diameter $=3 \mu \mathrm{m}$ ) in (a) $2 \mathrm{D}$; and (b) $3 \mathrm{D},(N=25)$, forming the text "GPC," using a distinct sphere-color for each character. In (b) virtual planes $z=-5 \mu \mathrm{m}, z=0$, and $z=+5 \mu \mathrm{m}$ intersect the centers-of-mass of the red, blue, and yellow-dyed spheres, respectively. of the red, blue, and yellow-dyed spheres, respectively.
Downloaded 10 Mar 2010 to 150.203 .243 .38 . Redistribution subject to AIP license or copyright; field given by ${ }^{19}$

phase function $\psi\left(u^{\prime}, v^{\prime}\right)$ onto this SPM. Since the director axis of this birefringent device is oriented at $45^{\circ}$ with $\hat{\boldsymbol{e}}_{v}$, the time-dependent reflected intensity pattern $I_{r}\left(u^{\prime}, v^{\prime} ; t\right)$ is proportional to the squared modulus of the complex reflected

$$
\begin{aligned}
\overrightarrow{\boldsymbol{E}}_{\boldsymbol{r}}\left(u^{\prime}, v^{\prime} ; t\right)= & E\left(u^{\prime}, v^{\prime} ; t\right)\left\{-i \sin \left[\psi\left(u^{\prime}, v^{\prime} ; t\right) / 2\right] \hat{\boldsymbol{e}}_{u}\right. \\
& \left.+\cos \left[\psi\left(u^{\prime}, v^{\prime} ; t\right) / 2\right] \hat{\boldsymbol{e}}_{v}\right\} .
\end{aligned}
$$

The bracketed terms of Eq. (2) denotes a polarization landscape that, in general, varies both in space and time. Depending on how we choose the space and the time dependence of $\phi\left(u^{\prime}, v^{\prime} ; t\right) \Rightarrow I\left(u^{\prime}, v^{\prime} ; t\right)$ and $\psi\left(u^{\prime}, v^{\prime} ; t\right)$, a set of operating modes for $4 \mathrm{D}$ optical manipulation can take place. Using a polarizing beam splitter (PBS), we have losslessly decomposed the orthogonally polarized components $(s$-pol and $p$-pol) of Eq. (2). The two components are finally projected to the sample along a common optical axis but with opposite $\boldsymbol{k}$ vectors. The system prealignment procedure requires the two projections to be superimposed transversely and focused onto respective planes that have a slight axial separation. The control over the individual relative strengths of polarization components associated with each trap is totally decoupled from each other, as seen from Eq. (2). In other words, using $N$ traps, each particle in a colloidal system under manipulation can be given a generally timedependent position vector $\overrightarrow{\boldsymbol{r}}_{n}(t)=x_{n}(t) \hat{\boldsymbol{e}}_{x}+y_{n}(t) \hat{\boldsymbol{e}}_{y}+z_{n}(t) \hat{\boldsymbol{e}}_{z}$ or its higher time derivatives (velocity, acceleration, and so on), as well. Functions $x_{n}(t)$ and $y_{n}(t)$ respectively possess direct proportionality to the SLM-controlled variables $u_{n}(t)$ and $v_{n}(t)$ in Eq. (1), and $z_{n}(t)$ is separately controlled by $\psi(u, v ; t)$ in Eq. (2). In Fig. 2, we illustrate one operation mode for creating full-space trajectories of simultaneously trapped polystyrene microbeads. ${ }^{23}$ The user-controlled positions $\left.\left(u_{n}, v_{n}\right)\right|_{n=1,2,3}$ of three traps enable us to interactively position three particles at the vertices of an imaginary triangle. We set $\left.\left(u_{n}, v_{n}\right)\right|_{n=1,2,3}$ to progressively vary taking coordinates that lie on a circle with $\psi=\psi\left(u^{\prime}, v^{\prime}\right)$, i.e., a temporally fixed polarization landscape. This effectively results in identical but out-of-phase time-varying position vectors for the particles, i.e.: $\overrightarrow{\boldsymbol{r}}_{1}(t)=\overrightarrow{\boldsymbol{r}}_{2}(t-T / 3)=\overrightarrow{\boldsymbol{r}}_{3}(t-2 T / 3)$, which trace out the 3D path shown in Fig. 2. $T$ denotes the time it takes for a sphere to traverse one cycle of the path.

The most general mode of operation is achieved by making the axial trap position an active control parameter like the transverse trap position rather than applying a fixed polarization landscape. To do so, we simply encode $\psi\left(u^{\prime}, v^{\prime} ; t\right)$ 

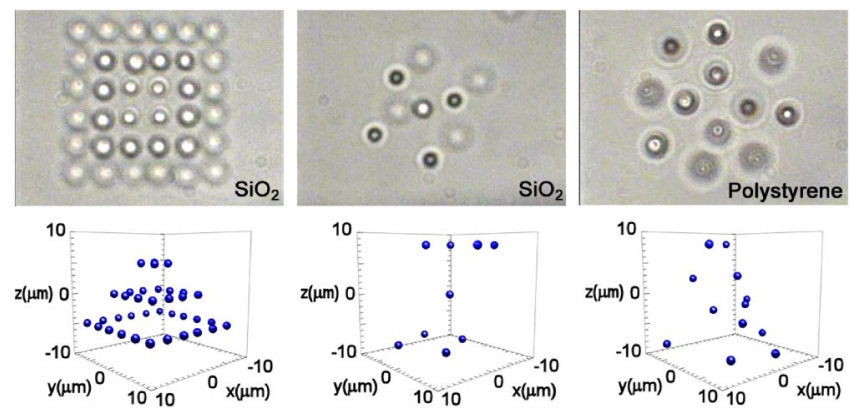

FIG. 4. (Color online) Optical trapping $\left(N_{\max }=36\right)$ and manipulation into arbitrary constellations of colloidal microspheres $\left(\mathrm{SiO}_{2}\right.$, diameter $=2.25 \mu \mathrm{m}$; polystyrene, diameter $=3 \mu \mathrm{m})$, top. $3 \mathrm{D}$ rendered view of the spheres' relative positions, bottom (see Ref. 23).

$$
\psi\left(u^{\prime}, v^{\prime} ; t\right)=\pi \sum_{n=1}^{N} \gamma_{n}(t) f_{n}\left[u^{\prime}-u_{n}^{\prime}(t), v^{\prime}-v_{n}^{\prime}(t)\right]
$$

Note that this is similar to $\phi(u, v)$ in Eq. (1), differing only by a factor $0 \leqslant \gamma_{n}(t) \leqslant 1$ inside the summation. By recalling that in Eq. (2) $\psi\left(u^{\prime}, v^{\prime}\right)$ determines the relative strengths of the orthogonal polarization components, one finds that the indexed weighting term $\gamma_{n}(t)$ enables axial control to be completely independent for each trap. The application of various operation modes has enabled us to perform genuinely real-time interactive optical manipulation of large arrays of particles (Figs. 3 and 4 ). ${ }^{23}$ We believe that these illustrations of interactively formed colloidal constellations and their user-defined dynamics have profound implications. It demonstrates the possibility of our system to allow access into exciting experiments involving arbitrarily patterned or dynamically driven systems of colloidal particles, including colloidal arrays created as analogs for modeling systems that are irrepressible or inaccessible within atomic and molecular domains. $^{2,3}$ For microbiologists, it serves as a noninvasive tool for manipulating cells into spatial configurations that may trigger variations in developmental features.

Over other alternatives, the GPC-based system offers several advantages. Further scaling of the real-time userinteractive manipulation to larger particle arrays is feasible since the GPC setup can in theory reconstruct fully dynamic arbitrary arrays with more than $90 \%$ efficiency requiring no computational overhead, which is compulsory in a DO-based approach. ${ }^{12}$ In the GPC-based system, trap resolution may be set equal to the SLM resolution and the lateral intensity distribution that form the traps stretch along the extent of the microscope field of view. Photon efficiency in a DO-based system depends on the spatial trap locations, and is compromised by the limited space-bandwidth product (SBWP) of the SLM, by the unwanted zero order and higher orders accompanying any desired diffraction pattern and by the strong spherical aberrations along the depth dimension associated with the use of high numerical aperture objective lens focusing. The GPC-based 4D manipulation system described here uses two key devices: a SLM to modulate phase and a SPM to modulate polarization of light. Nevertheless, unlike the complex task imposed by a DO-based method to synthesize a $3 \mathrm{D}$ light distribution on a single 2D phase-encoding device, separated transverse and axial trap control, which are not constrained by the SBWP, are assigned to the two devices, respectively. Finally, we emphasize that the simplicity of the binary phase modulation and the spatial polarization modulation scheme implies the future use of low-cost liquid crystal display technology.

Focusing on two attributes of an electromagnetic field, namely phase and polarization, we have formulated an energy-efficient way for crafting a single laser beam into real-time adjustable arrays of counterpropagating light fields, whose confining optical potentials enable the manipulation of multiple particles in 4D. The proposed system can, in due course, become an essential tool for unraveling issues that are yet to be understood in the physics of colloidal systems and the biology of microorganisms. It also has the potential for a range of technological applications where current optical manipulation schemes fall short.

This work has been funded by the European Science Foundation through the Eurocores-SONS program and partially by an internal grant awarded by Ris $\varnothing$ National Laboratory. The authors also acknowledge T. Hara and Y. Kobayashi of Hamamatsu Photonics for valuable discussions.

${ }^{1}$ D. G. Grier, Nature (London) 424, 810 (2003).

${ }^{2}$ M. M. Burns, J. M. Fournier, and J. A. Golovchenko, Phys. Rev. Lett. 63, 1233 (1989).

${ }^{3}$ M. M. Burns, J. M. Fournier, and J. A. Golovchenko, Science 249, 749 (1990).

${ }^{4}$ A. Simon and A. Libchaber, Phys. Rev. Lett. 68, 3375 (1992).

${ }^{5}$ A. T. O'Neil, I. MacVicar, L. Allen, and M. J. Padgett, Phys. Rev. Lett. 88, 053601 (2002).

${ }^{6}$ A. Terray, J. Oakley, and D. W. M. Marr, Science 296, 1841 (2002).

${ }^{7}$ M. P. MacDonald, G. C. Spalding, and K. Dholakia, Nature (London) 426, 421 (2003).

${ }^{8}$ J. Glückstad, Nature Mater. 3, 9 (2004).

${ }^{9}$ S. A. Tatarkova, A. E. Carruthers, and K. Dholakia, Phys. Rev. Lett. 89, 283901 (2002)

${ }^{10}$ J. P. Hoogenboom, D. L. J. Vossen, C. Faivre-Moskalenko, M. Dogerom, and A. van Blaaderen, Appl. Phys. Lett. 80, 4828 (2002).

${ }^{11}$ P. J. Rodrigo, R. L. Eriksen, V. R. Daria, and J. Glückstad, Opt. Express 10, 1550 (2002)

${ }^{12}$ J. Leach, G. Sinclair, P. Jordan, J. Courtial, M. J. Padgett, J. Cooper, and Z. J. Laczik, Opt. Express 12, 220 (2004).

${ }^{13}$ P. Jordan, H. Clare, L. Flendrig, J. Leach, J. Cooper, and M. Padgett, J. Mod. Opt. 51, 627 (2004).

${ }^{14}$ A. Terray, J. Oakley, and D. W. M. Marr, Appl. Phys. Lett. 81, 1555 (2002).

${ }^{15}$ R. L. Eriksen, V. R. Daria, and J. Glückstad, Opt. Express 10, 597 (2002).

${ }^{16}$ V. R. Daria, R. L. Eriksen, and J. Glückstad, J. Mod. Opt. 50, 1601 (2003).

${ }^{17}$ V. R. Daria, P. J. Rodrigo, and J. Glückstad, Appl. Phys. Lett. 84, 323 (2004).

${ }^{18}$ P. J. Rodrigo, V. R. Daria, and J. Glückstad, Opt. Lett. 19, 2270 (2004).

${ }^{19}$ R. L. Eriksen, P. C. Mogensen, and J. Glückstad, Opt. Commun. 187, 325 (2001).

${ }^{20}$ J. Glückstad, Opt. Commun. 130, 225-230 (1996).

${ }^{21}$ J. Glückstad, US Patent No. 6011874 (2000).

${ }^{22}$ J. Glückstad and P. C. Mogensen, Appl. Opt. 40, 268 (2001).

${ }^{23}$ Associated movie files can be found at http://www.ppo.dk/files/APLsupplementary. 